\title{
GW23-e1498 DURATION OF DUAL ANTIPLATELET THERAPY AND OUTCOMES AFTER LEFT MAIN PERCUTANEOUS CORONARY INTERVENTION
}

doi:10.1136/heartjnl-2012-302920l.26

Tao Hu, Hai-Chang Wang. Department of Cardiology, Xijing Hospital, Fourth Military Medical University, Xi'an 710032, China

Objectives Despite treatment recommendations for at least 12 months of dual antiplatelet therapy (DAPT) following drugeluting stent revascularisation, the optimal duration of DAPT after left main percutaneous coronary intervention remains controversial. We sought to evaluate differences in late safety outcomes relative to DAPT duration in patients treated with drug eluting stents in left main coronary artery.

Methods 216 patients undergoing successful primary percutaneous left main coronary intervention of dual antiplatelet therapy with aspirin $(100 \mathrm{mg} / \mathrm{d})$ and clopidogrel $(75 \mathrm{mg} / \mathrm{d})$ were eligible for enrolment in this randomised, double-blind, placebo-controlled trial from September 2008 to October 2011 at our institution. Patients were analysed according to continuation or discontinuation of DAPT at a 12-month landmark, excluding patients with events prior to the landmark, and outcomes were followed up to 36-months after stenting. Among patients who were event-free at 12 -months, clopidogrel was discontinued in 88 patients and was continued for longer than 36-months in 94 patients. The main outcome for our current analysis was Target Vessel Failure (TVF), defined as target vessel-related cardiac death or myocardial infarction and target vessel revascularisation. Secondary outcomes included stent thrombosis (ST). (Un) adjusted hazard ratios (HR) for TVF were calculated with Cox regression.

Results Through 3 years, risk-adjusted ischaemic event rates did not significantly differ between groups: 12 versus $\geq 36$ months: death (2.6\% vs $2.3 \%$ ), myocardial infarction (MI, $0.2 \%$ vs $1.0 \%$ ), and definite/probable stent thrombosis (ST, $0.2 \%$ vs $0 \%$ ). Composite events also did not statistically vary between DAPT durations. In multivariable analysis, 12-month versus longer DAPT duration was not associated with increased likelihood of thrombotic events at 3-year follow-up. Even after addition of identified independent predictors for TVF, adjusted TVF hazards were comparable. Major bleeding was negligible across groups.

Conclusions 12-month dual antiplatelet therapy seems to be feasible after left main percutaneous coronary intervention in Chinese patients. 